Research Article

\title{
A note on hyper-plane arrangements in $\mathbb{R}^{d}$
}

\author{
Nhattrieu Duong ${ }^{1}$, Moshe Idan $^{2}$, Rom Pinchasi ${ }^{3, *}$, Jason L. Speyer ${ }^{1}$ \\ ${ }^{1}$ Department of Mechanical and Aerospace Engineering, University of California, Los Angeles, USA \\ ${ }^{2}$ Faculty of Aerospace Engineering, Technion - Israel Institute of Technology, Haifa, Israel \\ ${ }^{3}$ Faculty of Mathematics, Technion - Israel Institute of Technology, Haifa, Israel
}

(Received: 12 May 2021. Accepted: 20 July 2021. Published online: 26 July 2021.)

(c) 2021 the authors. This is an open access article under the CC BY (International 4.0) license (www.creativecommons.org/licenses/by/4.0/).

\begin{abstract}
This note addresses hyper-plane arrangements in $\mathbb{R}^{d}$ and functions that are constant in the interior of each of the $d$-dimensional faces of the arrangement. We show that such a function $g$ can be expressed in a simple form using basis functions that are products of $d$ or less indicator functions of the open half-spaces bounded by the hyper-planes in the arrangement. Moreover, we present a simple and efficient algorithm that can be used to express $g$ as a linear combination of these basis functions.
\end{abstract}

Keywords: hyperplanes; hyperplane-arrangements; linear basis.

2020 Mathematics Subject Classification: 52C35.

\section{Introduction}

For hyper-planes $H_{1}, \ldots, H_{n}$ in $\mathbb{R}^{d}$ we denote by $\mathscr{A}$ the arrangement of the $n$ hyper-planes $H_{1}, \ldots, H_{n}$. Hyper-plane arrangements is a broad and well studied topic in mathematics with relations and applications to many other fields in mathematics and computer science $[5,8,9]$. The objects of $\mathscr{A}$ are all the possible intersections of hyper-planes and open half-spaces defined by $H_{1}, \ldots, H_{n}$. Of particular interest to us will be the $d$-dimensional faces of the arrangement $\mathscr{A}$. These are in fact the connected components of $\mathbb{R}^{d} \backslash \cup_{i=1}^{n} H_{i}$.

We recall the function sign : $\mathbb{R} \rightarrow \mathbb{R}$ that is equal to 1 for every positive number and is equal to -1 for negative numbers. We artificially define sign (0) to be -1 . As will be elaborated below, the value of sign (0) will not be important for us at all in the sequel.

In this note we address functions $g: \mathbb{R}^{d} \rightarrow \mathbb{R}$ that can be expressed in terms of the $n$ sign functions sign $\left(\left\langle x, v_{i}\right\rangle-c_{i}\right)$, $1 \leq i \leq n$, where $v_{i} \in \mathbb{R}^{n}$ and $c_{i} \in \mathbb{R}$ are some given vectors and constants, respectively. Such functions were encountered in the study of estimation and control of linear systems forced by Cauchy [3, 4, 6, 7] and Laplacian [1,2] noises. When derived, these functions $g$ have a very complex form that leads to numerical difficulties in applications. The goal of this note is to propose an alternative and simpler representation of those functions. In addition, it will show a simple and fast algorithm to construct this representation. In this paper we will not care about the values of the function $g$ at points $x$ for which $\left\langle x, v_{i}\right\rangle-c_{i}=0$ for some $1 \leq i \leq n$. This is the reason why the value of sign (0) will not be of any importance in this paper. For convenience we set sign $(0)=-1$.

It will be more convenient to work with the functions $\sigma_{i}(x)=\frac{1}{2}\left[\operatorname{sign}\left(\left\langle x, v_{i}\right\rangle-c_{i}\right)+1\right]$. Notice that $\sigma_{i}(x)$ is the indicator function of the open half-space $H_{i}^{+}=\left\{x \in \mathbb{R}^{d} \mid\left\langle x, v_{i}\right\rangle>c_{i}\right\}$. That is, $\sigma_{i}(x)=1$ for every $x$ in $H_{i}^{+}$and is equal to 0 otherwise. Observe that a function $g$ can be expressed in terms of the functions $\operatorname{sign}\left(\left\langle x, v_{i}\right\rangle-c_{i}\right)$ if and only if it can be expressed in terms of $\sigma_{1}, \ldots, \sigma_{n}$.

Because each of the functions $\sigma_{1}, \ldots, \sigma_{n}$ is constant in every $d$-dimensional face of $\mathscr{A}$, then so are the functions $g$ that we study in this note.

It is easy to see that also the other way around is true. That is, given $H_{1}, \ldots, H_{n}$, any function $g: \mathbb{R}^{d} \backslash \cup_{i=1}^{n} H_{i} \rightarrow \mathbb{R}$ that is constant in every $d$-dimensional face of $\mathscr{A}$ can be expressed in terms of $\sigma_{1}, \ldots, \sigma_{n}$. To prove this observe that it is enough to address functions $g$ that are equal to 1 for every $x$ in some $d$-dimensional face $F$ of $\mathscr{A}$ and are equal to 0 otherwise. Let $I_{n}=\{1, \ldots, n\}$ and $I \subset I_{n}$ be the set of all indices $i$ such that $H_{i}$ supports a $(d-1)$-dimensional facet of $F$. Then $F$ is equal to the intersection of all half-spaces containing $F$ and bounded by $H_{i}$ for some $i \in I$. Let $I_{a}$ be the set of all indices $i \in I$ such that $\sigma_{i}(x)=1$ for every $x \in F$. Let $I_{b}=I \backslash I_{a}$. Then $g=\prod_{i \in I_{a}} \sigma_{i} \cdot \prod_{i \in I_{b}}\left(1-\sigma_{i}\right)$. Consequently, any function 
$g: \mathbb{R}^{d} \backslash \cup_{i=1}^{n} H_{i} \rightarrow \mathbb{R}$ that is constant in every $d$-dimensional face of $\mathscr{A}$ can be written as a linear combination of products of $n$ or less of $\sigma_{1}, \ldots, \sigma_{n}$.

The main result in this note is the following improvement that is also tight.

Theorem 1.1. Let $\mathscr{A}$ be a hyper-plane arrangement of $n$ affine hyper-planes $H_{1}, \ldots, H_{n}$ in $\mathbb{R}^{d}$ defined by $H_{i}=\left\{x \mid\left\langle x, v_{i}\right\rangle=c_{i}\right\}$, where $x \in \mathbb{R}^{d}, v_{i} \in \mathbb{R}^{d}$ is normal to $H_{i}$, and $c_{i} \in \mathbb{R}$. For every $1 \leq i \leq n$ let $\sigma_{i}$ denote the indicator function of the open halfspace $\left\{x \mid\left\langle x, v_{i}\right\rangle>c_{i}\right\}$ bounded by $H_{i}$. Let $g$ be any function that is constant in the interior of every d-dimensional face in $\mathscr{A}$. Then there is a linear combination of products of $d$ or less of the functions $\sigma_{i}$ that is equal to g at any point in $\mathbb{R}^{d} \backslash \cup_{i=1}^{n} H_{i}$.

Given a hyper-plane arrangement $\mathscr{A}$ of $n$ affine hyper-planes $H_{1}, \ldots, H_{n}$ in $\mathbb{R}^{d}$ and a function $g: \mathbb{R}^{d} \backslash \cup_{i=1}^{n} H_{i} \rightarrow \mathbb{R}$ that is constant on every $d$-dimensional face of $\mathscr{A}$, Theorem 1.1 tells us that we can write $g$ as a linear combination of products of $d$ or less of the functions $\sigma_{1}, \ldots, \sigma_{n}$. Specifically, let $I \subset I_{n}$ be a subset of $I_{n}$ with cardinality $|I| \leq d$ and denote by $\sigma_{I}$ the product $\prod_{i \in I} \sigma_{i}$. When $I$ is the empty set, we define $\sigma_{\emptyset}=1$. Theorem 1.1 implies that the function $g$ can be expressed as

$$
g=\sum_{|I| \leq d} a_{I} \sigma_{I}
$$

for all $I \subset I_{n}$ with $|I| \leq d$ with some coefficients $a_{I}$.

The number of such possible products $\sigma_{I}$ and thus terms in the sum of (1) is equal to $N=\sum_{i=0}^{d}\left(\begin{array}{c}n \\ i\end{array}\right)$. This raises the question of an efficient computation of the $N$ coefficients $a_{I}$ in (1). In Section 3 we provide an algorithm that given the function $g$ computes these coefficients with running time of $O\left(2^{d}\left(\begin{array}{l}n \\ d\end{array}\right)\right)$. That is, number of operations in the algorithm is $O\left(2^{d}\left(\begin{array}{c}n \\ d\end{array}\right)\right)$, where calling to the function $g$ is considered as one operation.

\section{Proof of Theorem 1.1}

We start with a preliminary result that will be used to prove the main theorem presented next. When stated separately, not within the problem addressed in this note, its statement and proof can be greatly simplified, without hampering its generalization.

Lemma 2.1. Let $\Delta_{d}$ be a $d$-simplex in $\mathbb{R}^{d}$. Let $H_{1}, \ldots, H_{d+1}$ be the $d+1$ affine hyper-planes supporting the facets of $\Delta_{d}$. For $i=1, \ldots, d+1$, let $\sigma_{i}$ be the indicator function of the closed half-space bounded by $H_{i}$ and containing $\Delta_{d}$. Then

$$
\prod_{i=1}^{d+1}\left(1-\sigma_{i}\right)=0 .
$$

Proof. Assume, without loss of generality, that $0 \in \Delta_{d}$. For $i=1, \ldots, d+1$ we write $H_{i}$ as $H_{i}=\left\{x \mid\left\langle x, v_{i}\right\rangle=c_{i}\right\}$, where $v_{i} \in \mathbb{R}^{d}$ (orthogonal to $H_{i}$ ) is chosen such that $c_{i}>0$. Then $\Delta_{d}=\left\{x \mid \forall 1 \leq i \leq d+1,\left\langle x, v_{i}\right\rangle \leq c_{i}\right\}$. Observe that the statement of the Lemma 2.1 is equivalent to saying that there is no vector $u \in \mathbb{R}^{d}$ such that $\left\langle u, v_{i}\right\rangle>c_{i}$ for every $1 \leq i \leq d+1$. Assume to the contrary that there is such a vector $u$. Then for every $\alpha>0$ and every $1 \leq i \leq d+1$ we have $\left\langle-\alpha u, v_{i}\right\rangle=-\alpha\left\langle u, v_{i}\right\rangle<-\alpha c_{i}<0<c_{i}$. In other words, $-\alpha u \in \Delta_{d}$ for every $\alpha>0$. This is impossible as $\Delta_{d}$ is bounded.

We can now proceed to the proof of Theorem 1.1. Observe that in order to prove Theorem 1.1 it is enough to consider functions $g$ that are indicator functions of $d$-dimensional faces in $\mathscr{A}$.

Let $F$ be a $d$-dimensional face in $\mathscr{A}$ and let $g$ be the indicator function of $F$. Let $I \subseteq I_{n}$ denote the set of indices $i$ such that $H_{i}$ supports $F$ at a facet of dimension $d-1$. Let $I_{a}$ and $I_{b}$ be a partition of $I$ into two parts such that if $i \in I_{a}$, then $F \subset\left\{x \mid\left\langle x, v_{i}\right\rangle>c_{i}\right\}$ and if $i \in I_{b}$, then $F \subset\left\{x \mid\left\langle x, v_{i}\right\rangle<c_{i}\right\}$. Observe that $F$ is equal to the intersection of all open half-spaces containing $F$ that are bounded by some hyper-plane $H_{i}$ where $i \in I$. Therefore, the function

$$
\tilde{g}=\prod_{i \in I_{a}} \sigma_{i} \cdot \prod_{i \in I_{b}}\left(1-\sigma_{i}\right)
$$

is equal to $g$ at any point not in $\cup_{i=1}^{n} H_{i}$.

If the cardinality $|I|$ of $I$ is smaller than or equal to $d$, we are done because $\tilde{g}$ can clearly be written as a linear combination of products of $|I|$ or less of the indicator functions $\sigma_{1}, \ldots, \sigma_{n}$. If the cardinality of $I$ is larger than $d$, then $\tilde{g}$ can still be written as a linear combination of products of the indicator functions $\sigma_{1}, \ldots, \sigma_{n}$, however the number of terms in each product may exceed $d$. Therefore, Theorem 1.1 will follow if we can show that the product of every $d+1$ of the indicator functions $\sigma_{1}, \ldots, \sigma_{n}$ is equal, on $\mathbb{R}^{d} \backslash \cup_{i=1}^{n} H_{i}$, to a linear combination of products of $d$ or less of the indicator functions $\sigma_{1}, \ldots, \sigma_{n}$. 
We prove this by induction on $d$. The basis of the induction is the case $d=1$. In this case we have two indicator functions, say $\sigma_{1}$ and $\sigma_{2}$. We would like to consider the function $\sigma_{1} \sigma_{2}$ and express it as a linear combination of products of zero or one of the functions $\sigma_{1}$ and $\sigma_{2}$.

This could easily be left to the reader, but for completeness we bring the simple analysis here. For $i=1,2$ there exists $x_{i}$ such that the function $\sigma_{i}$ is either the indicator function of $\left\{x \mid x<x_{i}\right\}$ or of $\left\{x \mid x>x_{i}\right\}$. Without loss of generality assume that $x_{1} \leq x_{2}$. We consider four possible cases.

Case 1. $\sigma_{1}$ is the indicator function of $\left\{x \mid x<x_{1}\right\}$ and $\sigma_{2}$ is the indicator function of $\left\{x \mid x<x_{2}\right\}$. In this case $\sigma_{1} \sigma_{2}=\sigma_{1}$.

Case 2. $\sigma_{1}$ is the indicator function of $\left\{x \mid x<x_{1}\right\}$ and $\sigma_{2}$ is the indicator function of $\left\{x \mid x>x_{2}\right\}$. In this case $\sigma_{1} \sigma_{2}=0$.

Case 3. $\sigma_{1}$ is the indicator function of $\left\{x \mid x>x_{1}\right\}$ and $\sigma_{2}$ is the indicator function of $\left\{x \mid x<x_{2}\right\}$. In this case $\sigma_{1} \sigma_{2}$ is equal to the function $\sigma_{1}+\sigma_{2}-1$.

Case 4. $\sigma_{1}$ is the indicator function of $\left\{x \mid x>x_{1}\right\}$ and $\sigma_{2}$ is the indicator function of $\left\{x \mid x>x_{2}\right\}$. In this case $\sigma_{1} \sigma_{2}$ is equal to the function $\sigma_{2}$.

This concludes the case $d=1$ being the basis of induction.

For $d>1$ we consider two possible cases:

Case 1. $k+1$ of the vectors $v_{1}, \ldots, v_{d+1}$ are linearly dependent for some $1 \leq k<d$. Without loss of generality, assume that $v_{1}, \ldots, v_{k+1}$ are linearly dependent. By a possible rotation of $\mathbb{R}^{d}$, we can assume that $\operatorname{span}\left\{v_{1}, \ldots, v_{k+1}\right\} \subseteq \operatorname{span}\left\{e_{1}, \ldots, e_{k}\right\}$, where $e_{1}, \ldots, e_{k}$ are the first $k$ elements of the standard basis of $\mathbb{R}^{d}$. Let $P: \mathbb{R}^{d} \rightarrow \mathbb{R}^{k}$ be the projection on the first $k$ coordinates of $\mathbb{R}^{d}$. In $\mathbb{R}^{k}$, for every $1 \leq i \leq k+1$ we define $H_{i}^{\prime}=\left\{x \in \mathbb{R}^{k} \mid\left\langle P\left(v_{i}\right), x\right\rangle=c_{i}\right\}$ and let $\sigma_{i}^{\prime}: \mathbb{R}^{k} \rightarrow \mathbb{R}$ be the indicator function of $\left\{x \in \mathbb{R}^{k} \mid\left\langle P\left(v_{i}\right), x\right\rangle>c_{i}\right\}$. Observe that for every $x \in \mathbb{R}^{d}$ and $1 \leq i \leq k+1$,

$$
\sigma_{i}(x)=\sigma_{i}^{\prime}(P(x)) .
$$

Because $k<d$, we can apply the induction hypothesis for dimension $k$ and conclude that $\prod_{i=1}^{k+1} \sigma_{i}^{\prime}$ is equal to a linear combination of products of $k$ or less of $\sigma_{1}^{\prime}, \ldots, \sigma_{k+1}^{\prime}$. Because of (3) it follows that $\prod_{i=1}^{k+1} \sigma_{i}$ is equal to a linear combination of products of $k$ or less of $\sigma_{1}, \ldots, \sigma_{k+1}$. Consequently,

$$
\prod_{i=1}^{d+1} \sigma_{i}=\prod_{i=1}^{k+1} \sigma_{i} \cdot \prod_{i=k+2}^{d+1} \sigma_{i}
$$

is equal to a linear combination of products of $d$ or less of $\sigma_{1}, \ldots, \sigma_{d+1}$.

Case 2. every set of $d$ vectors from $v_{1}, \ldots, v_{d+1}$ is linearly independent. We split into two possible subcases.

Case 2a. $\cap_{i=1}^{d+1} H_{i}=\emptyset$. In this case $H_{1}, \ldots, H_{d+1}$ are the $d+1$ affine hyper-planes supporting the facets of the $d$-simplex $\Delta_{d}$ whose vertices are $v_{j}=\cap_{i=1, i \neq j}^{d+1} H_{i}$ for $1 \leq j \leq d+1$. We may assume, without loss of generality, that $0 \in \Delta_{d}$. Let $I=\{1, \ldots, d+1\}$. Let $I_{a}$ and $I_{b}$ be a partition of $I$ into two parts such that if $i \in I_{a}$, then $c_{i}<0$, and if $i \in I_{b}$, then $c_{i}>0$. Then

$$
\prod_{i \in I_{a}} \sigma_{i} \cdot \prod_{i \in I_{b}}\left(1-\sigma_{i}\right)
$$

is the indicator function of the interior of $\Delta_{d}$. Applying (2) of Lemma 2.1 yields

$$
\prod_{i \in I_{a}}\left(1-\sigma_{i}\right) \cdot \prod_{i \in I_{b}} \sigma_{i}=0
$$

which proves the theorem for this case.

Case 2b. $\cap_{i=1}^{d+1} H_{i} \neq \emptyset$. In this case $\cap_{i=1}^{d+1} H_{i}$ is a single point, because $v_{1}, \ldots, v_{d}$ are linearly independent. Without loss of generality we assume that this single point is 0 . Consequently, $c_{i}=0$ for $1 \leq i \leq d+1$. Let $\alpha_{1}, \ldots, \alpha_{d+1}$ be real numbers, not all zero, such that $\sum_{i=1}^{d+1} \alpha_{i} v_{i}=0$. Notice that in this case $\alpha_{i} \neq 0$ for all $1 \leq i \leq d+1$, because every set of $d$ vectors from $v_{1}, \ldots, v_{d+1}$ is linearly independent.

Define $I_{a}=\left\{1 \leq i \leq d+1 \mid \alpha_{i}>0\right\}$ and $I_{b}=\left\{1 \leq i \leq d+1 \mid \alpha_{i}<0\right\}$. Notice that $I_{a}$ and $I_{b}$ form a partition of $\{1, \ldots, d+1\}$. We claim that

$$
\prod_{i \in I_{a}} \sigma_{i} \cdot \prod_{i \in I_{b}}\left(1-\sigma_{i}\right)=0 .
$$

Observe that once (4) is established we are done, as (4) implies that $\prod_{i=1}^{d+1} \sigma_{i}$ is a linear combination of products of $d$ or less of $\sigma_{1}, \ldots, \sigma_{d+1}$.

To prove (4), notice that the contrary assumption is that there exists a vector $u$ such that for every $i \in I_{a}$ we have $\left\langle u, v_{i}\right\rangle>0$ and for every $i \in I_{b}$ we have $\left\langle u, v_{i}\right\rangle<0$. It follows now from the definition of $I_{a}$ and $I_{b}$ that for every $1 \leq i \leq d+1$ 
we have $\alpha_{i}\left\langle u, v_{i}\right\rangle>0$. This is a contradiction as

$$
\sum_{i=1}^{d+1} \alpha_{i}\left\langle u, v_{i}\right\rangle=\left\langle u, \sum_{i=1}^{d+1} \alpha_{i} v_{i}\right\rangle=\langle u, 0\rangle=0 .
$$

\section{Computational aspects}

Assume we are given a hyper-plane arrangement $\mathscr{A}$ of $n$ affine hyper-planes $H_{1}, \ldots, H_{n}$ in $\mathbb{R}^{d}$ and a function $g$ that is constant on each of the $d$-dimensional faces of $\mathscr{A}$. For every $i=1, \ldots, n$ we let $\sigma_{i}$ be the indicator function of a open halfspace bounded by $H_{i}$ (we may choose any of the two). In this section we provide an algorithm of running time $O\left(2^{d}\left(\begin{array}{l}n \\ d\end{array}\right)\right)$ that produces the representation of $g$ as a linear combination of products of $d$ or less of $\sigma_{1}, \ldots, \sigma_{n}$.

We start with a preliminary observation that we will need.

Observation 3.1. Assume $H_{1}, \ldots, H_{d}$ are $d$ hyper-planes in $\mathbb{R}^{d}$, passing through the origin, with normal vectors that are linearly independent. Then $H_{1}, \ldots, H_{d}$ partition $\mathbb{R}^{d}$ into $2^{d}$ regions and the horizontal hyper-plane $\left\{x_{d}=0\right\}$ must avoid at least two of them. Moreover, if $\left\{x_{d}=0\right\}$ is not parallel to any intersection of $d-1$ or less of $H_{1}, \ldots, H_{d}$, then the horizontal hyper-plane must avoid exactly two of the $2^{d}$ regions.

Proof. Perhaps the easiest way to see this is to perform a linear transformation on $\mathbb{R}^{d}$ such that $H_{1}, \ldots, H_{d}$ coincide with the $d$ axis-parallel hyper-planes through the origin. Then $H_{1}, \ldots, H_{d}$ partition $\mathbb{R}^{d}$ into $2^{d}$ region $F_{1}, \ldots, F_{2^{d}}$ that correspond to the $2^{d}$ different sign patterns of the $d$ coordinates.

The linear transformation we performed takes the horizontal hyper-plane $\left\{x_{d}=0\right\}$ to some hyper-plane $H$ with a normal vector $v$. Observe that the condition that $H$ is not parallel to any intersection of $d-1$ or less of $H_{1}, \ldots, H_{d}$ is equivalent to that no coordinate of $v$ is equal to 0 .

There are at least two of the regions $F_{1}, \ldots, F_{2^{d}}$ (and exactly two if the coordinates of $v$ are all different from 0 ) whose coordinates sign patterns either always agree with the sign pattern of the coordinates of $v$, or always disagree with the sign pattern of the coordinates of $v$. These are exactly the regions avoided by $H$.

We will first assume that the arrangement $\mathscr{A}$ is in general position in the sense that no $d+1$ hyper-planes from $H_{1}, \ldots, H_{n}$ share a common point. For reasons that will become clear shortly, we assume without loss of generality that no face in $\mathscr{A}$ is horizontal, i.e., is parallel to the hyper-plane $\left\{x_{d}=0\right\}$. This can be achieved by a generic rotation of $\mathscr{A}$.

We start by computing all the $\left(\begin{array}{l}n \\ d\end{array}\right)$ vertices of $\mathscr{A}$. This can easily be done in time $O\left(d^{3}\left(\begin{array}{l}n \\ d\end{array}\right)\right)$ (we do not try to optimize here as long as we are at least as fast as $\left.O\left(2^{d}\left(\begin{array}{l}n \\ d\end{array}\right)\right)\right)$, simply by considering every subset of $d$ of hyper-planes from $H_{1}, \ldots, H_{n}$ and then solving a system of $d$ linear equations in $d$ variables.

It follows from Observation 3.1 that every vertex in $\mathscr{A}$ is the unique lowest point of a unique $d$-dimensional face in $\mathscr{A}$. Indeed, at every vertex $X$ of $\mathscr{A}$ apply Observation 3.1, where $H_{1}, \ldots, H_{d}$ are taken to be the $d$ hyper-planes meeting at $X$ and let $H$ in Observation 3.1 be the horizontal hyper-plane through $X$, that is, $H$ is the hyper-plane through $X$ parallel to $\left\{x_{d}=0\right\}$.

Theorem 1.1 tells us that any function $g$ that is constant on every $d$-dimensional face of $\mathscr{A}$ can be written as a linear combination of products of $d$ or less of $\sigma_{1}, \ldots, \sigma_{n}$, given in (1). Notice that because $H_{1}, \ldots, H_{n}$ are in general position this implies that the $N=\sum_{i=0}^{d}\left(\begin{array}{c}n \\ i\end{array}\right)$ products of $d$ or less of $\sigma_{1}, \ldots, \sigma_{n}$ form in fact a basis for the space of all functions $g$ that are constant on every $d$-dimensional face of $\mathscr{A}$. This is because the number of $d$-dimensional faces in any arrangement of $n$ hyper-planes in general position is precisely $N$ (see $[5,8,9]$ ). Although we will not use the fact that the products of $d$ or less of $\sigma_{1}, \ldots, \sigma_{n}$ are linearly independent, it is useful to observe this fact in order to understand better the proof.

We will use, however, the following simple observation. We claim that even if we replace any $\sigma_{i}$ by $\left(1-\sigma_{i}\right)$ in any of the products of $d$ or less of $\sigma_{1}, \ldots, \sigma_{n}$ we will still remain with a basis for the space of all functions $g$ that are constant on every $d$-dimensional face of $\mathscr{A}$. To see this, let $I$ be a set of $k$ indices from $I_{n}$, where $1 \leq k \leq d$ (the case $k=0$ is trivial). Let $I^{\prime} \subset I$ be any subset of $I$. We would like to show that we can replace $\prod_{i \in I} \sigma_{i}$ by $\prod_{i \in I^{\prime}}\left(1-\sigma_{i}\right) \cdot \prod_{i \in I \backslash I^{\prime}} \sigma_{i}$ and remain with a basis for the space of all functions $g$ that are constant on every $d$-dimensional face of $\mathscr{A}$. This is indeed true because $\prod_{i \in I^{\prime}}\left(1-\sigma_{i}\right) \cdot \prod_{i \in I \backslash I^{\prime}} \sigma_{i}$ is equal to $\pm \prod_{i \in I} \sigma_{i}$ plus a linear combination of products of $k-1$ or less of $\sigma_{1}, \ldots, \sigma_{n}$. This fact can now be used to prove by induction on $k$ that the replacement of any $\sigma_{i}$ by $\left(1-\sigma_{i}\right)$ does not change the linear span of these products.

We are given a function $g$ that is constant on every $d$-dimensional face of $\mathscr{A}$ and we would like to find the representation of it, that surely exists because of Theorem 1.1, as in (1). In particular, we want to find the $N$ coefficients $a_{I}$ given the values of $g$ in the $N d$-dimensional faces of $\mathscr{A}$. 
For every $I \subset I_{n}$ such that $|I|=d$ we consider the point $X_{I}$ that is the intersection of all the $d$ hyper-planes $H_{i}$ where $i \in I$. As we observed, $X_{I}$ is the unique lowest point of a unique $d$-dimensional face that we denote by $F_{I}$. Let $I^{\prime} \subset I$ be the set of all indices $i \in I$ such that $\sigma_{i}$ is equal to 0 on $F_{I}$. Define $\sigma_{I}^{\prime}=\prod_{i \in I^{\prime}}\left(1-\sigma_{i}\right) \cdot \prod_{i \in I \backslash I^{\prime}} \sigma_{i}$. Observe that $\sigma_{I}^{\prime}$ is equal to 0 at every point below $X_{I}$. Moreover, $\sigma_{I}^{\prime}$ is equal to 0 in all the $d$-dimensional faces that have $X_{I}$ as a vertex, except for $F_{I}$ where $\sigma_{I}^{\prime}$ is equal to 1 .

As we already noticed, $g$ can be written also as a linear combination of $\sigma_{I}^{\prime}$ for $|I|=d$ and $\sigma_{I}$ for $|I|<d$. Let $a_{I}^{\prime}$ for $|I| \leq d$ be the coefficients such that $g=\sum_{|I|=d} a_{I}^{\prime} \sigma_{I}^{\prime}+\sum_{|I|<d} a_{I}^{\prime} \sigma_{I}$. Let $H$ be a horizontal hyper-plane that is lower than the lowest vertex of $\mathscr{A}$. Notice that $H$ intersects precisely all the $d$-dimensional faces that do not have a lowest point. Notice also that $\sum_{|I|=d} a_{I}^{\prime} \sigma_{I}^{\prime}$ is equal to 0 at every point on $H$ simply because $H$ is lower than all the vertices in $\mathscr{A}$. Therefore, on $H$ we have $g=\sum_{|I|<d} a_{I}^{\prime} \sigma_{I}$. We therefore consider the $(d-1)$-dimensional arrangement of $H \cap H_{1}, \ldots, H \cap H_{n}$ in the $d-1$ dimensional space $H$. We use induction and find the representation of $g=\sum_{|I|<d} a_{I}^{\prime} \sigma_{I}$ on $H$ with running time $T(d-1)$ that we will analyze later (we will show that $T(d)=O\left(2^{d}\left(\begin{array}{l}n \\ d\end{array}\right)\right)$ ). Having found the coefficients $a_{I}^{\prime}$ for $|I|<d$ we proceed to finding the coefficients $a_{I}^{\prime}$ for $|I|=d$.

Fix $I \subset I_{n}$ such that $|I|=d$. We show how to find $a_{I}^{\prime}$. The point $X_{I}$ is the intersection of the $d$ hyper-planes $H_{i}$, where $i \in I$. By Observation 3.1, these hyper-planes partition $\mathbb{R}^{d}$ into $2^{d} d$-dimensional regions and therefore, $X_{I}$ is a vertex of precisely $2^{d} d$-dimensional faces of $\mathscr{A}$. Denote these $d$-dimensional faces by $F_{1}, \ldots, F_{2^{d}}$ and recall that $F_{I}$ is one of these faces.

Let $S_{I}$ be the function $\prod_{i \in I^{\prime}}\left(2\left(1-\sigma_{i}\right)-1\right) \cdot \prod_{i \in I \backslash I^{\prime}}\left(2 \sigma_{i}-1\right)$. To understand the simple meaning of $S_{I}$ observe that $2 \sigma_{i}-1$ is equal to 1 in the half-space where $\sigma_{i}=1$ and is equal to -1 in the half-space where $\sigma_{i}=0$ (the same is true for $\left(2\left(1-\sigma_{i}\right)-1\right)$ with the change of role of the two half-spaces bounded by $H_{i}$ ). Therefore, $S_{I}$ is equal to 1 in $F_{I}$ (where $\left(1-\sigma_{i}\right)=1$ for all $i \in I^{\prime}$ and $\sigma_{i}=1$ for all $\left.i \in I \backslash I^{\prime}\right)$. Moreover $S_{I}$ changes sign every time we cross one of the hyper-planes $H_{i}$, where $i \in I$.

We claim that

$$
a_{I}^{\prime}=\sum_{i=1}^{2^{d}} g\left(F_{i}\right) S_{I}\left(F_{i}\right) .
$$

Notice that evaluating $a_{I}^{\prime}$ in this way takes time $2^{d}$ (that is, $2^{d}$ calls to the function $g$ ).

To prove (6) recall that $g=\sum_{|J|=d} a_{J}^{\prime} \sigma_{J}^{\prime}+\sum_{|J|<d} a_{J}^{\prime} \sigma_{J}$. Hence, (6) is restated a

$$
a_{I}^{\prime}=\sum_{|J|=d} \sum_{i=1}^{2^{d}} a_{J}^{\prime} \sigma_{J}^{\prime}\left(F_{i}\right) S_{I}\left(F_{i}\right)+\sum_{|J|<d} \sum_{i=1}^{2^{d}} a_{J}^{\prime} \sigma_{J}\left(F_{i}\right) S_{I}\left(F_{i}\right) .
$$

Observe that we have $\sum_{i=1}^{2^{d}} a_{I}^{\prime} \sigma_{I}^{\prime}\left(F_{i}\right) S_{I}\left(F_{i}\right)=a_{I}^{\prime} \sigma_{I}^{\prime}\left(F_{I}\right) S_{I}\left(F_{I}\right)=a_{I}^{\prime}$. This is because $\sigma_{I}^{\prime}$ is equal to 0 on all the faces $F_{1}, \ldots, F_{2^{d}}$ except for $F_{I}$ on which $\sigma_{I}^{\prime}$ is equal to 1 . Therefore, in order to complete the proof of (6) it is enough to examine the sums in (7) with $J \neq I$ and show that $\sum_{i=1}^{2^{d}} a_{J}^{\prime} \sigma_{J}^{\prime}\left(F_{i}\right) S_{I}\left(F_{i}\right)=0$ for $|J|=d$ and $\sum_{i=1}^{2^{d}} a_{J}^{\prime} \sigma_{J}\left(F_{i}\right) S_{I}\left(F_{i}\right)=0$ for $|J|<d$.

Let $J \subset I_{n},|J|=d$, and $J \neq I$. Because $|I|=d$, it must be that $I \backslash J$ is not empty. Choose arbitrarily some $q \in I \backslash J$. For every face $F_{i}$ the value $\sigma_{J}^{\prime}\left(F_{i}\right)$ is either 1 or 0 . If $\sigma_{J}^{\prime}\left(F_{i}\right)=0$ for all $1 \leq i \leq 2^{d}$, we are done. For every face $F_{i}$ with $\sigma_{J}^{\prime}\left(F_{i}\right)=1$ consider the unique face $F_{i^{\prime}}$ such that $F_{i}$ and $F_{i^{\prime}}$ share a common facet on the hyper-plane $H_{q}$. Observe that for $F_{i^{\prime}}$ it is also true that $\sigma_{J}^{\prime}\left(F_{i^{\prime}}\right)=1$ because $q \notin J$. However $S_{I}\left(F_{i}\right)=-S_{I}\left(F_{i^{\prime}}\right)$. Therefore, in $\sum_{i=1}^{2^{d}} a_{J}^{\prime} \sigma_{J}^{\prime}\left(F_{i}\right) S_{I}\left(F_{i}\right)$ the terms $i$ and $i^{\prime}$ for which $\sigma_{J}^{\prime}\left(F_{i}\right)=\sigma_{J}^{\prime}\left(F_{i^{\prime}}\right)=1$ cancel the contributions of each other, while the other terms are identically zero because their respective $\sigma_{J}^{\prime}=0$. Consequently, $\sum_{i=1}^{2^{d}} a_{J}^{\prime} \sigma_{J}^{\prime}\left(F_{i}\right) S_{I}\left(F_{i}\right)=0$. A similar argument as above holds when $|J|<d$ to yield that $\sum_{i=1}^{2^{d}} a_{J}^{\prime} \sigma_{J}\left(F_{i}\right) S_{I}\left(F_{i}\right)=0$. This concludes the proof of equation (6).

We can therefore find all the coefficients $a_{I}^{\prime}$ for $|I|=d$ in time $O\left(2^{d}\left(\begin{array}{c}n \\ d\end{array}\right)\right)$, as there are $\left(\begin{array}{c}n \\ d\end{array}\right)$ subsets $I$ of $I_{n}$ with $|I|=d$. To summarize, we found all the coefficients $a_{I}^{\prime}$ in the representation of $g$ as $g=\sum_{|I|=d} a_{I}^{\prime} \sigma_{I}^{\prime}+\sum_{|I|<d} a_{I}^{\prime} \sigma_{I}$ in time $O\left(2^{d}\left(\begin{array}{l}n \\ d\end{array}\right)\right)+T(d-1)$.

We are not done yet because we need to find the representation of $g$ as $g=\sum_{|I| \leq d} a_{I} \sigma_{I}$. In order to do this we recall that every $\sigma_{I}^{\prime}$ for $|I|=d$ is a product of $d$ terms each of which is equal either to $\sigma_{i}$ or to $\left(1-\sigma_{i}\right)$ for some $i \in I$. We can therefore write it as linear sum of at most $2^{d}$ products of $d$ or less of $\sigma_{i}$ where $i \in I$. This requires an additional work of time $O\left(2^{d}\left(\begin{array}{l}n \\ d\end{array}\right)\right)$. Then we get a representation of $g$ as a linear sum of at most

$$
2^{d}\left(\begin{array}{l}
n \\
d
\end{array}\right)+\left(\begin{array}{c}
n \\
d-1
\end{array}\right)+\left(\begin{array}{c}
n \\
d-2
\end{array}\right)+\ldots+\left(\begin{array}{l}
n \\
0
\end{array}\right)
$$

products of $d$ or less of $\sigma_{1}, \ldots, \sigma_{n}$. With additional work of $O\left(2^{d}\left(\begin{array}{l}n \\ d\end{array}\right)\right)$ we can gather similar terms and get the required representation of $g$ as in (1). 
In order to analyze the running time $T(d)$ we observe that $T(d)=T(d-1)+O\left(2^{d}\left(\begin{array}{l}n \\ d\end{array}\right)\right)$. This easily implies $T(d)=O\left(2^{d}\left(\begin{array}{l}n \\ d\end{array}\right)\right)$.

We have thus proved that the desired representation of $g$ can be computed in time $O\left(2^{d}\left(\begin{array}{l}n \\ d\end{array}\right)\right)$ when the arrangement $\mathscr{A}$ is in general position. We next show how to conclude from here an algorithm with the same running time also when $\mathscr{A}$ may not be in general position. What we do is perturb by just a little bit the hyper-planes $H_{1}, \ldots, H_{n}$ and define $g$ to be 0 (although it could take arbitrary values just as well) in the newly created $d$-dimensional faces. More precisely, we first take sample points from every face in $\mathscr{A}$ before the perturbation. Then we perturb $\mathscr{A}$. We find which new $d$-dimensional faces correspond to the old ones. This can be done very quickly, as we just need to find the new $d$-dimensional faces to which each of the old sample points belong. Now that we have the perturbed hyper-plane arrangement that is in general position and the new function $g$, we apply our algorithm and the representation that we get for the new $g$ will work also for the original one.

A disadvantage of the above method for arrangements $\mathscr{A}$ that are not in a general position is that the representation we obtain for $g$ has more terms in (1) than the number of $d$-dimensional faces in $\mathscr{A}$. Effectively it means that the basis we use to represent $g$ is larger than needed and one can find a smaller one, i.e., $g$ could be fully represented as in (1) with the number of terms that equals to the number of $d$-dimensional faces in $\mathscr{A}$. In the next section we present an algorithm that finds such a minimum-dimensional basis and shows how to find the coefficients of a representation of $g$ with this basis.

\section{The non-general-position case}

Theorem 4.1. Let $\mathscr{A}$ be a hyper-plane arrangement of $n$ affine hyper-planes $H_{1}, \ldots, H_{n}$ in $\mathbb{R}^{d}$. For every $1 \leq i \leq n$ let $\sigma_{i}$ denote the indicator function of one of the two open half-spaces bounded by $H_{i}$. Let $\mathscr{G}$ be the linear vector space consisting of all functions $g$ that are constant in the interior of every $d$-dimensional face in $\mathscr{A}$. Then there is a basis for $\mathscr{G}$ that consists of products of $d$ or less of the functions $\sigma_{i}$.

Remark. The size of the basis guaranteed in Theorem 4.1 is clearly equal to the dimension of $\mathscr{G}$, that is, the number of $d$-dimensional faces in $\mathscr{A}$.

Proof. We prove the theorem by induction on $d$. For $d=1$ there is nothing to prove because in this case the theorem is equivalent to Theorem 1.1.

Assume the theorem is true for dimension $d-1$ and we prove it for dimension $d$. Given $d$ is fixed, we prove the theorem by induction on $n$. The theorem is clearly true for $n=1$ because in this case $\mathscr{A}$ consists of just two $d$-dimensional faces, that is, the two open half-spaces bounded by $H_{1}$. Then a basis for $\mathscr{G}$ is $\left\{1, \sigma_{1}\right\}$ and this is regardless of the choice of the indicator function $\sigma_{1}$.

Assume the theorem is true for $n-1$ and we prove it for $n$. We would like to find a basis for $\mathscr{G}$ in which every element is a product of $d$ or less of $\sigma_{1}, \ldots, \sigma_{n}$. Consider the hyper-plane arrangement $\tilde{\mathscr{A}}$ of the $n-1$ hyper-planes $H_{1}, \ldots, H_{n-1}$. By induction hypothesis, there is a basis whose elements are products of $d$ or less of $\sigma_{1}, \ldots, \sigma_{n-1}$ for the linear space $\tilde{\mathscr{G}}$ of all the functions that are constant on the interior of every $d$-dimensional face of $\tilde{\mathscr{A}}$. Denote this basis by $a_{1}, \ldots, a_{k}$, where $k$ is the dimension of $\tilde{\mathscr{G}}$.

Consider now the hyper-plane $H_{n}$. For $i=1, \ldots, n-1$ denote by $H_{i}^{\prime}$ the intersection $H_{i} \cap H_{n}$. Therefore, $H_{i}^{\prime}$ is a hyperplane of dimension $d-1$ in $H_{n}$. Let $\mathscr{A}^{\prime}$ denote the hyper-plane arrangement of $H_{1}^{\prime}, \ldots, H_{n-1}^{\prime}$ inside $H_{n}$. Let $\mathscr{G}^{\prime}$ be the linear space of all functions that are constant on every $(d-1)$-dimensional face of $\mathscr{A}^{\prime}$. For $i=1, \ldots, n-1$ let $\sigma_{i}^{\prime}$ denote the restriction of $\sigma_{i}$ to $H_{n}$. Notice that $\sigma_{i}^{\prime}$ is the indicator function of one of the open half-spaces of $H_{n}$ bounded by $H_{i}^{\prime}$. By the induction hypothesis, there is a basis for $\mathscr{G}^{\prime}$ whose elements are products of $d-1$ or less of $\sigma_{1}^{\prime}, \ldots, \sigma_{n-1}^{\prime}$. Denote the elements of this basis by $b_{1}^{\prime}, \ldots, b_{\ell}^{\prime}$, where $\ell$ is the dimension of $\mathscr{G}^{\prime}$. For $j=1, \ldots, \ell$ let $I_{j}$ be the set of indices from $\{1, \ldots, n-1\}$ such that $b_{j}^{\prime}=\prod_{i \in I_{j}} \sigma_{i}^{\prime}$.

We observe that the number of $d$-dimensional faces in $\mathscr{A}$ is equal to the number of $d$-dimensional faces in $\tilde{\mathscr{A}}$ plus the number of $(d-1)$-dimensional faces in $\mathscr{A}^{\prime}$. In other words, the dimension of $\mathscr{G}$ is equal to $k+\ell$, which is the sum of the dimension of $\tilde{\mathscr{G}}$ and the dimension of $\mathscr{G}^{\prime}$.

We are now ready to define the desired basis for $\mathscr{G}$. For $j=1, \ldots, \ell$, considering the subsets $I_{j}$ used to define $b_{j}^{\prime}$ above, define $b_{j}=\prod_{i \in I_{j}} \sigma_{i}$. We claim that $B=\left\{a_{1}, \ldots, a_{k}\right\} \cup\left\{\sigma_{n} b_{1}, \ldots, \sigma_{n} b_{\ell}\right\}$ is a basis for $\mathscr{G}$. We remark that once we prove $B$ is a basis for $\mathscr{G}$ we are done, as every member of $B$ is a product of $d$ or less of $\sigma_{1}, \ldots, \sigma_{n}$. Moreover, to conclude that $B$ is indeed a basis for $\mathscr{G}$ it is enough to show that it spans $\mathscr{G}$, because $|B|$ is equal to $k+\ell$, the dimension of $\mathscr{G}$.

In order to show that $B$ spans $\mathscr{G}$ it is enough to show that the indicator function of every $d$-dimensional face in $\mathscr{A}$ can be written as a linear combination of members of $B$. Let $C$ be a $d$-dimensional face of $\mathscr{A}$. If none of the facets of $C$ is 
supported by $H_{n}$, then $C$ is also a $d$-dimensional face in $\tilde{\mathscr{A}}$ and therefore the indicator function of $C$ can be written as a linear combination of $a_{1}, \ldots, a_{k}$.

Assume therefore that $H_{n}$ supports a facet of $C$. There is a unique cell in $\tilde{A}$ that contains $C$. We denote this cell by $\tilde{C}$. In fact $C$ is equal to the intersection of $\tilde{C}$ with one of the two open half-spaces bounded by $H_{n}$.

Let $C^{\prime}=H_{n} \cap \tilde{C}$. $C^{\prime}$ is a $(d-1)$-dimensional face in $\mathscr{A}^{\prime}$. Therefore, the indicator function of $C^{\prime}$ can be written as $\sum_{j=1}^{\ell} \beta_{j} b_{j}^{\prime}$ for some coefficients $\beta_{1}, \ldots, \beta_{\ell}$. Now consider the function $g=\sum_{j=1}^{\ell} \beta_{j} b_{j}$. Notice that $g$ is constant on every $d$ dimensional face of $\mathscr{A}$. Moreover, because the restriction of $g$ to $H_{n}$ is equal to $\sum_{j=1}^{\ell} \beta_{j} b_{j}^{\prime}$, that is, to the indicator function of $C^{\prime}$, then $g$ must be equal to 1 on $\tilde{C}$ and must be equal to 0 on every other $d$-dimensional face of $\tilde{\mathscr{A}}$ whose interior is intersected by $H_{n}$.

Consider now the function $\sigma_{n} g$. This function is constant on every $d$-dimensional face of $\tilde{\mathscr{A}}$ except for $\tilde{C}$. Indeed, $\sigma_{n} g$ is constant on every $d$-dimensional face of $\tilde{\mathscr{A}}$ whose interior is not intersected by $H_{n}$. It is equal to 0 on every $d$-dimensional face of $\tilde{\mathscr{A}}$ whose interior is intersected by $H_{n}$ except for $\tilde{C}$. The face $\tilde{C}$ is a union of two $d$-dimensional faces in $\mathscr{A}$, namely, $C$ and $\tilde{C} \backslash C$. The function $\sigma_{n} g$ is equal to 1 on one of $C$ and $\tilde{C} \backslash C$ and is equal to 0 on the other.

Let $f$ be the function that is equal to $\sigma_{n} g$ except that $f$ is equal to 0 on $\tilde{C}$. Because $f$ is in $\tilde{\mathscr{G}}$, we can write $f=\sum_{j=1}^{k} \alpha_{j} a_{j}$ for some coefficients $\alpha_{1}, \ldots, \alpha_{k}$. Now, the function $\sigma_{n} g-f$ is the indicator function of either $C$ or $\tilde{C} \backslash C$, the one for which $\sigma_{n} g$ is equal to 1 . If $\sigma_{n} g-f$ is the indicator function of $C$, then we are done because

$$
\sigma_{n} g-f=\sum_{j=1}^{\ell} \beta_{j} \sigma_{n} b_{j}-\sum_{j=1}^{k} \alpha_{j} a_{j} .
$$

If $\sigma_{n} g-f$ is the indicator function of $\tilde{C} \backslash C$, then write the indicator function of $\tilde{C}$ as $\sum_{j=1}^{k} \gamma_{j} a_{j}$. Then the indicator function of $C$ is equal to

$$
\sum_{j=1}^{k} \gamma_{j} a_{j}-\left(\sigma_{n} g-f\right)=\sum_{j=1}^{k}\left(\gamma_{j}+\alpha_{j}\right) a_{j}-\sum_{j=1}^{\ell} \beta_{j} \sigma_{n} b_{j}
$$

and we are done again.

\section{Acknowledgments}

This work was supported in part by the National Science Foundation under grant numbers NSF/ENG/ECCS-BSF 1607502 and 1934467 as well as the NSF-BSF ECCS grant number 2019639. Rom Pinchasi acknowledges the financial support from the Ministry of Educational and Science of the Russian Federation in the framework of MegaGrant No. 075-15-2019-1926.

\section{References}

[1] N. Duong, J. L. Speyer, M. Idan, Laplace Controller for Linear Scalar Systems, 27th Mediterranean Conference on Control and Automation (MED), Akko, Israel, July 2019.

[2] N. Duong, J. L. Speyer, J. Yoneyama, M. Idan, Laplace Estimator for Linear Scalar Systems, 57th IEEE Conference on Decision and Control, Miami, FL, USA, December 2018.

[3] J. Fernández, J. L. Speyer, M. Idan, Stochastic estimation for two-state linear dynamic systems with additive Cauchy noises, IEEE Trans. Automat. Control 60 (2015) 3367-3372.

[4] J. Fernández, J. L. Speyer, M. Idan, Stochastic control for linear systems with additive Cauchy noises, IEEE Trans. Automat. Control 60 (2015) 3373-3378.

[5] J. E. Goodman, J. O’Rourke, C. D. Tóth (Eds.), Handbook of Discrete and Computational Geometry, Third Edition, CRC Press, Boca Raton, 2018.

[6] M. Idan, J. L. Speyer, Multivariate Cauchy estimator with scalar measurent and process noises, SIAM J. Control Optim. 52 (2014) 1108-1141.

[7] M. Idan, J. L. Speyer, Characteristic Function Approach to Smoothing of Linear Scalar Systems with Additive Cauchy Noises, 27th Mediterranean Conference on Control and Automation (MED), Akko, Israel, July 2019.

[8] R. Stanley, Enumerative Combinatorics, Vol. 1, 2nd Edition, Chapter 3.11: Hyperplane Arrangements, Cambridge University Press, Cambridge, 2011.

[9] T. Zaslavsky, Facing Up to Arrangements: Face-Count Formulas for Partitions of Space by Hyperplanes, Mem. Amer. Math. Soc., Providence, 1975. 